\title{
Linking new and old concepts: inflammation meets the Warburg phenomenon in pulmonary arterial hypertension
}

\author{
Todd M. Kolb • Rachel L. Damico • Paul M. Hassoun
}

Published online: 7 July 2011

(C) Springer-Verlag 2011

Pulmonary arterial hypertension (PAH) is a devastating syndrome characterized by endothelial cell dysfunction, hypertrophy and proliferation of medial smooth muscle cells, and adventitial inflammation and fibrosis $[1,2]$. Although the worldwide prevalence remains unknown, a recent report estimates the prevalence at 15 cases per million persons and the annual incidence at 2.4 cases per million persons in the French population [3]. Despite significant advances in diagnosis and therapeutics, mortality remains unacceptably high, with 3-year survival estimated at only $67 \%$ [4]. Survival in patients with $\mathrm{PAH}$ associated with systemic sclerosis (SSc) is even worse [5], and PAH is currently the second leading cause of death in this population [6]. Current therapies have been effective at reducing the morbidity associated with $\mathrm{PAH}$ and may have demonstrated some mortality benefit in specific World Health Organization class I groups such as idiopathic PAH. Historically, patients with SSc-associated PAH have obtained less benefit from modern therapies for reasons that remain the active subject of intensive investigations.

Regardless of the etiology of PAH, currently available therapies are based on intensive efforts over the past 40 years to understand the roles of various mediators in the maintenance of pulmonary vascular tone through regulation of smooth muscle cell and endothelial cell function [2]. More recently, research efforts have been directed toward understanding the role of inflammation in the pathogenesis of PAH. Following the recognition of

T. M. Kolb · R. L. Damico • P. M. Hassoun $(\bowtie)$

Division of Pulmonary and Critical Care Medicine

and Department of Medicine,

Johns Hopkins University School of Medicine,

1830 East Monument Street, 5th Floor,

Baltimore, MD 21205, USA

e-mail: phassoun@jhmi.edu complex inflammatory cell infiltrates surrounding plexiform lesions in human PAH [7], there has been a dramatic expansion in the amount of literature focused on the potential mechanistic relevance of inflammation in PAH (Fig. 1). This work has contributed greatly to an evolving paradigm of PAH as an inflammatory disease as recently reviewed [1]. A growing number of cytokines and growth factors have been demonstrated to be increased in serum from PAH patients. Mechanistic hypotheses have focused on a link between inflammation and endothelial cell dysfunction, endovascular thrombosis, smooth muscle cell proliferation and contraction, and adventitial fibrosis/ remodeling.

Most recently, new data have implicated alterations in pulmonary arterial smooth muscle cell (PASMC) glucose metabolism in the vascular remodeling of PAH [8]. PASMC isolated from animal models of PAH demonstrate phenotypic alterations which include changes in mitochondrial membrane potential, potassium flux, and sensitivity to apoptotic death. Importantly, suppression of these phenotypic changes via forced glucose oxidation and mitochondrial respiration is protective. Furthermore, experimentally induced right ventricular (RV) afterload results in a shift in myocardial energy metabolism characterized by increased glucose uptake, increased glycolysis (Gly), and decreased glucose oxidation (GO) $[9,10]$. Pyruvate dehydrogenase kinase (PDK), the enzyme which inhibits conversion of pyruvate to acetyl-CoA for oxidative metabolism, appears to play a key role in this shift $[9,10]$. Furthermore, dichloroacetate, a PDK inhibitor, has been shown to reverse RV hypertrophy in animal models of pulmonary hypertension [11]. Taken together, these studies highlight a potential link between altered glucose metabolism and the pathogenesis of PAH. Similar metabolic changes have long been recognized to contribute to the pathogenesis of cancer since 


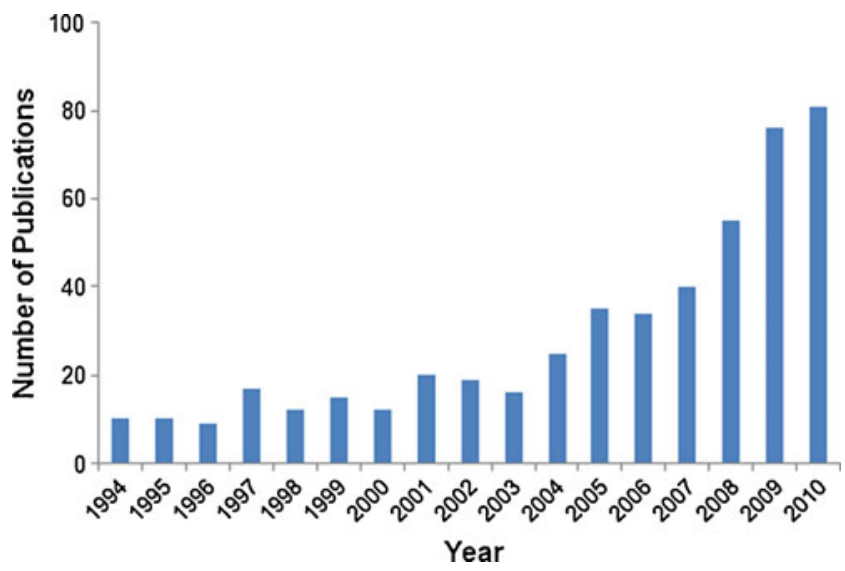

Fig. 1 Number of yearly publications since 1994 obtained through a PubMed search when using the index words "inflammation" and "pulmonary hypertension"

their initial description in tumors by Otto Warburg in 1956 [12]. The relative contribution of altered glucose metabolism and mitochondrial bioenergetics in the vasculopathy and $\mathrm{RV}$ response in human PAH remains to be determined.

Until now, mechanistic connections between changes in glucose metabolism and the evolving paradigm of PAH as an inflammatory disease were absent. In this issue, Sutendra et al. provide compelling evidence for a potential mechanistic link between these two evolving concepts in PAH pathogenesis [13]. The study supports a hypothesis linking the inflammatory cytokine tumor necrosis factor alpha $(\mathrm{TNF} \alpha)$ to PASMC proliferation and inhibition of apoptosis through pyruvate dehydrogenase (PDH), a critical enzyme required for GO. In this scheme (Fig. 2), increased $\mathrm{TNF} \alpha$ in the vascular microenvironment inhibits PDH in a PDK-dependent manner. At the level of the mitochondrion, PDH inhibition results in decreased cellular respiration and mitochondrial reactive oxygen species (ROS) generation. As a consequence of this change in mitochondrial energetics, there are alterations in the redox- and voltage-sensitive mitochondrial transition pore resulting in hyperpolarization of the mitochondria and a subsequent increase in the threshold for release of mitochondrial sequestered pro-apoptotic molecules. At the cellular level, potassium equilibrium is altered, resulting in membrane depolarization, calcium influx, and ultimately PASMC contraction and/or proliferation. In total, the inhibition of PDH by TNF $\alpha$ induces a smooth muscle cell phenotype characterized by increased contractility, proliferation, and reduced apoptosis, events all linked to altered GO. At the tissue level, these changes would be reflected by increased pulmonary vascular tone and medial remodeling.

To support this hypothesis, the authors demonstrate that recombinant $\mathrm{TNF} \alpha$ significantly reduced PDH activity in human PASMC in vitro [13]. In addition, the TNF $\alpha$ inhibitor etanercept prevented and reversed RV hypertrophy and pulmonary vascular remodeling in a well characterized

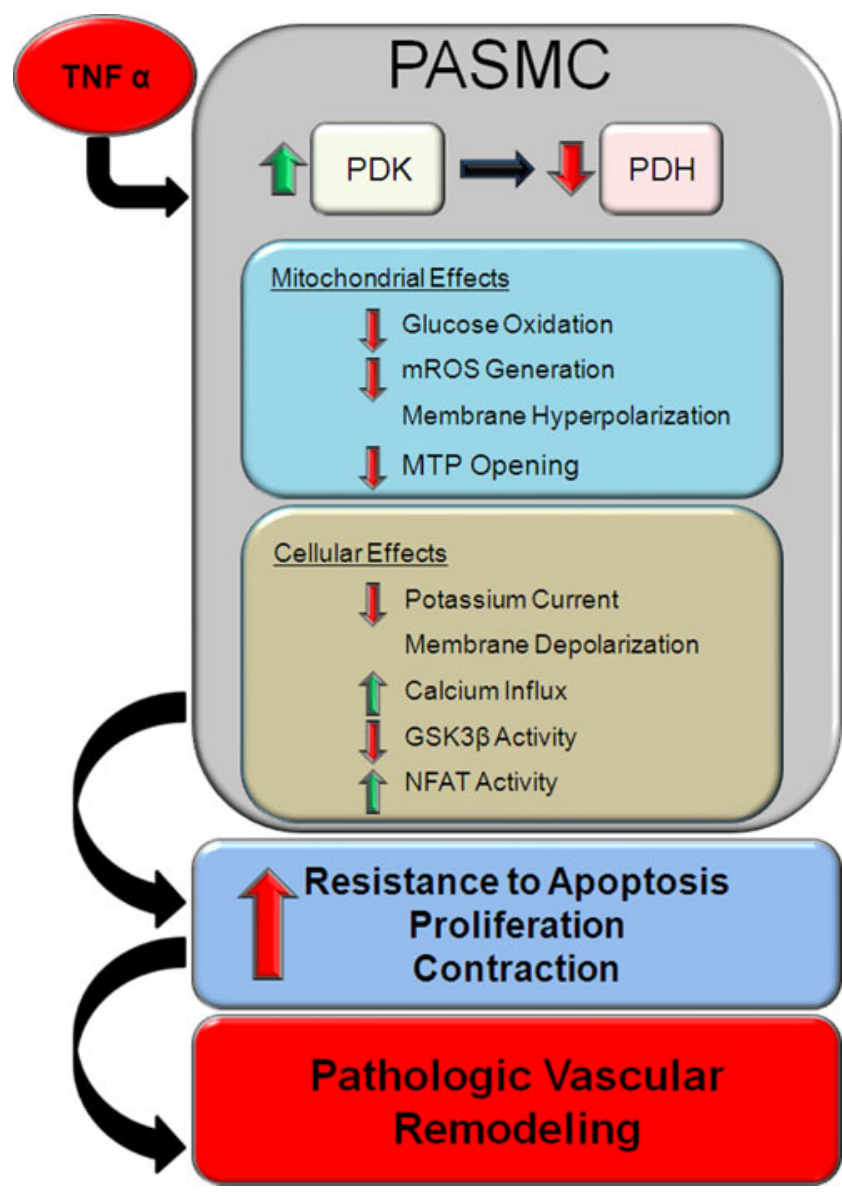

Fig. 2 Proposed mechanisms of the effects of TNF $\alpha$ on PASMC phenotype in PAH. Via suppression of PDH, TNF $\alpha$ alters mitochondrial metabolism resulting in decreased mROS and membrane hyperpolarization which suppresses apoptosis. Changes in mROS also modify $\mathrm{K}_{\mathrm{v}}$ channel activity altering calcium homeostasis and activity of GSK $3 \beta$ and NFAT. These changes increase proliferation and decrease apoptosis of PASMC during pathologic vascular remodeling. $P D K$ pyruvate dehydrogenase kinase, $m R O S$ mitochondrial reactive oxygen species, MTP mitochondrial transition pore, GSK3 $\beta$ glycogen synthase kinase $3 \beta$, NFAT nuclear factor of activated T cells

monocrotaline (MCT)-induced rat model of PAH. Reversal of vascular remodeling with etanercept was associated with reduced proliferation and increased apoptosis of PASMC in vivo. Furthermore, $T$ cell-deficient rats were more resistant to MCT-induced PAH implicating T cell-derived factors in the remodeling. Finally, human PASMC cultured in the presence of activated CD8 $+\mathrm{T}$ cells demonstrated mitochondrial hyperpolarization, decreased mROS, lowered $\mathrm{K}_{\mathrm{v}^{-}}$ dependent potassium current, increased intracellular calcium, and activation of the transcription factor nuclear factor of activated $\mathrm{T}$ lymphocytes. These changes were not observed in PASMC cultured with unstimulated CD8+ $\mathrm{T}$ cells and were abrogated with a TNF $\alpha$-specific antibody. Thus, the authors conclude that $\mathrm{TNF} \alpha$, potentially derived from activated CD8+ T cells, can inhibit PDH activity in PASMC, yielding a proliferative and 
apoptosis-resistant phenotype. More importantly, these studies provide a mechanistic rationale for the use of anti-inflammatory agents as potential therapeutic strategies in PAH.

Alterations in glucose metabolism have previously been described in other inflammatory conditions, including the metabolic syndrome [14] and sepsis [15]. A transition of energy substrate utilization towards increased Gly is well described in left ventricular hypertrophy and failure [16] and TNF $\alpha$ has recently been implicated in this transition through inhibition of PDK4 [17]. As described above, similar metabolic changes occur in the RV during pulmonary hypertension and in animal models of pulmonary hypertension, RV remodeling can be reversed by inhibiting PDK and increasing PDH activity. This observation raises the theoretical possibility of added clinical benefit for $\mathrm{TNF} \alpha$-specific therapies to suppress $\mathrm{RV}$ remodeling and/or maintain maximally efficient myocardial energy substrate utilization during the development of pulmonary hypertension.

Conceptually, it is extremely attractive to consider a scenario where targeted anti-inflammatory therapies might have the potential to reverse pulmonary and/or myocardial remodeling in patients with PAH. Importantly, $\mathrm{TNF} \alpha$ inhibitors are currently available in clinical practice, are used routinely in patients with auto-immune disorders, and have very favorable profiles regarding safety and efficacy data in long-term treatment protocols. A recent case series of SSc patients showed that long-term use of etanercept for synovitis was successful in greater than $80 \%$ of patients, with only few adverse effects even after more than 5 years of therapy [18].

However, several clinical observations reinforce the fact that additional human data are necessary prior to advocating large-scale clinical trials of TNF $\alpha$ inhibitors. As Sutendra et al. [13] point out, in other autoimmune disease (like rheumatoid arthritis) where $\mathrm{TNF} \alpha$ levels are routinely elevated, the frequency of PAH is lower than that seen in patients with SSc. In addition, although TNF $\alpha$ expression is increased in SSc [19], trials of nonspecific antiinflammatory therapies in connective tissue diseaseassociated PAH have been somewhat disappointing. While cyclophosphamide and glucocorticoid treatment produced symptomatic benefit in PAH associated with systemic lupus erythematosus or mixed connective tissue disorder, there was no evidence of benefit in patients with SSc-PAH [20]. $\mathrm{TNF} \alpha$ levels were not routinely measured in that trial and it is possible that directed anti-inflammatory therapy in a subgroup of SSc patients with increased serum TNF $\alpha$ levels may be more successful. There is evidence that serum $\mathrm{TNF} \alpha$ levels may be higher in patients with limited than diffuse SSc [21]; the former is more frequently associated with PAH.
While the impressive reversal of PAH in the MCT rat model reported in the present study is intriguing, enthusiasm must be somewhat tempered, as the application of findings in the rodent MCT model of PAH to humans has often been very limited. It also remains unclear why the impressive findings in the MCT model were not recapitulated with chronic hypoxia. Several lines of evidence support the finding that hypoxia is an inflammatory condition; treatment strategies that provide benefit across multiple different animal models may ultimately prove most promising in human clinical trials. Finally, PASMC isolated from PAH patients maintain their altered phenotype ex vivo, suggesting that some phenotypic differences are autonomous of the PAH microenvironment from which the cells were derived. It is possible that PAH PASMC may produce sufficient TNF $\alpha$ to generate an autocrine loop and suppress PDH. The contribution of both resident vascular cells and recruited inflammatory cells as sources of TNF $\alpha$ deserves further evaluation. Nonetheless, the ability of etanercept to normalize the PAH PASMC phenotype in humans remains to be demonstrated. Clearly, human trials will be necessary to confirm the impressive results demonstrated in the present study and clarify their clinical applicability.

On the whole, Sutendra et al. [13] provide evidence for a provocative link between excessive inflammation and altered PASMC glucose metabolism in the mechanism of pulmonary vascular remodeling in PAH. These types of studies provide an important step in our evolving approach to understanding the pathogenesis of this complex disorder and may ultimately offer a fresh approach to the design of therapeutic agents with disease-modifying properties and mortality benefits. Borrowing from the experience of Otto Warburg in the 1950s, this study may be an important step in transforming care for PAH patients from symptom management to a longer lifespan.

\section{References}

1. Hassoun PM, Mouthon L, Barbera JA, Eddahibi S, Flores SC, Grimminger F, Jones PL, Maitland ML, Michelakis ED, Morrell NW et al (2009) Inflammation, growth factors, and pulmonary vascular remodeling. J Am Coll Cardiol 54:S10 S19

2. Zaiman A, Fijalkowska I, Hassoun PM, Tuder RM (2005) One hundred years of research in the pathogenesis of pulmonary hypertension. Am J Respir Cell Mol Biol 33:425-431

3. Humbert M, Sitbon O, Chaouat A, Bertocchi M, Habib G, Gressin V, Yaici A, Weitzenblum E, Cordier JF, Chabot F et al (2006) Pulmonary arterial hypertension in France: results from a national registry. Am J Respir Crit Care Med 173:1023-1030

4. Humbert M, Sitbon O, Chaouat A, Bertocchi M, Habib G, Gressin V, Yaici A, Weitzenblum E, Cordier JF, Chabot F et al (2010) Survival in patients with idiopathic, familial, and anorexigenassociated pulmonary arterial hypertension in the modern management era. Circulation 122:156-163 
5. Chung L, Liu J, Parsons L, Hassoun PM, McGoon M, Badesch DB, Miller DP, Nicolls MR, Zamanian RT (2010) Characterization of connective tissue disease-associated pulmonary arterial hypertension from REVEAL: identifying systemic sclerosis as a unique phenotype. Chest 138:1383-1394

6. Steen VD, Medsger TA (2007) Changes in causes of death in systemic sclerosis, 1972-2002. Ann Rheum Dis 66:940-944

7. Tuder RM, Groves B, Badesch DB, Voelkel NF (1994) Exuberant endothelial cell growth and elements of inflammation are present in plexiform lesions of pulmonary hypertension. Am J Pathol 144:275-285

8. Sutendra G, Bonnet S, Rochefort G, Haromy A, Folmes KD, Lopaschuk GD, Dyck JR, Michelakis ED (2010) Fatty acid oxidation and malonyl-coa decarboxylase in the vascular remodeling of pulmonary hypertension. Sci Transl Med 2:44ra58

9. Piao L, Marsboom G, Archer SL (2010) Mitochondrial metabolic adaptation in right ventricular hypertrophy and failure. J Mol Med 88:1011-1020

10. Piao L, Fang YH, Cadete VJ, Wietholt C, Urboniene D, Toth PT, Marsboom G, Zhang HJ, Haber I, Rehman J et al (2019) The inhibition of pyruvate dehydrogenase kinase improves impaired cardiac function and electrical remodeling in two models of right ventricular hypertrophy: Resuscitating the hibernating right ventricle. J Mol Med 88:47-60

11. Michelakis ED, McMurtry MS, Wu XC, Dyck JR, Moudgil R, Hopkins TA, Lopaschuk GD, Puttagunta L, Waite R, Archer SL (2002) Dichloroacetate, a metabolic modulator, prevents and reverses chronic hypoxic pulmonary hypertension in rats: Role of increased expression and activity of voltage-gated potassium channels. Circulation 105:244-250

12. Warburg O (1956) On respiratory impairment in cancer cells. Science 124:269-270
13. Sutendra G, Dromparis P, Bonnet S, Haromy A, McMurtry MS, Bleackley RC, Michelakis ED (2011) Pyruvate dehydrogenase inhibition by the inflammatory cytokine TNFa contributes to the pathogenesis of pulmonary arterial hypertension. J Mol Med (this issue)

14. Salmenniemi U, Ruotsalainen E, Pihlajamaki J, Vauhkonen I, Kainulainen S, Punnonen K, Vanninen E, Laakso M (2004) Multiple abnormalities in glucose and energy metabolism and coordinated changes in levels of adiponectin, cytokines, and adhesion molecules in subjects with metabolic syndrome. Circulation 110:3842-3848

15. Vary TC (1991) Increased pyruvate dehydrogenase kinase activity in response to sepsis. Am J Physiol 260:E669-E674

16. Depre C, Rider MH, Hue L (1998) Mechanisms of control of heart glycolysis. Eur J Biochem/FEBS 258:277-290

17. Alvarez-Guardia D, Palomer X, Coll T, Davidson MM, Chan TO, Feldman AM, Laguna JC, Vazquez-Carrera M (2010) The p65 subunit of nf-kappab binds to pgc-1alpha, linking inflammation and metabolic disturbances in cardiac cells. Cardiovasc Res $87: 449-458$

18. Lam GK, Hummers LK, Woods A, Wigley FM (2007) Efficacy and safety of etanercept in the treatment of scleroderma-associated joint disease. J Rhematol 34:1636-1637

19. Pendergrass SA, Hayes E, Farina G, Lemaire R, Farber HW, Whitfield ML, Lafyatis R (2010) Limited systemic sclerosis patients with pulmonary arterial hypertension show biomarkers of inflammation and vascular injury. PLoS One 5:e12106

20. Sanchez O, Sitbon O, Jais X, Simonneau G, Humbert M (2006) Immunosuppressive therapy in connective tissue diseasesassociated pulmonary arterial hypertension. Chest 130:182-189

21. Ingegnoli F, Trabattoni D, Saresella M, Fantini F, Clerici M (2003) Distinct immune profiles characterize patients with diffuse or limited systemic sclerosis. Clin Immunol 108:21-28 